\title{
THE D'ALEMBERT-LAGRANGE PRINCIPLE FOR GRADIENT THEORIES AND BOUNDARY CONDITIONS
}

\author{
H. GOUIN \\ Université d'Aix-Marseille, 13397 Marseille Cedex 20, France \\ E-mail: henri.gouin@univ-cezanne.fr \\ Dedicated to Prof. Antonio M. Greco
}

\begin{abstract}
Motions of continuous media presenting singularities are associated with phenomena involving shocks, interfaces or material surfaces. The equations representing evolutions of these media are irregular through geometrical manifolds. A unique continuous medium is conceptually simpler than several media with surfaces of singularity. To avoid the surfaces of discontinuity in the theory, we transform the model by considering a continuous medium taking into account more complete internal energies expressed in gradient developments associated with the variables of state. Nevertheless, resulting equations of motion are of an higher order than those of the classical models: they lead to non-linear models associated with more complex integration processes on the mathematical level as well as on the numerical point of view. In fact, such models allow a precise study of singular zones when they have a non negligible physical thickness. This is typically the case for capillarity phenomena in fluids or mixtures of fluids in which interfacial zones are transition layers between phases or layers between fluids and solid walls. Within the framework of mechanics for continuous media, we propose to deal with the functional point of view considering globally the equations of the media as well as the boundary conditions associated with these equations. For this aim, we revisit the d'Alembert-Lagrange principle of virtual works which is able to consider the expressions of the works of forces applied to a continuous medium as a linear functional value on a space of test functions in the form of virtual displacements. At the end, we analyze examples corresponding to capillary fluids. This analysis brings us to numerical or asymptotic methods avoiding the difficulties due to singularities in simpler -but with singularities- models.
\end{abstract}

\section{Introduction}

A mechanical problem is generally studied through force interactions between masses located in material points: this Newton point of view leads together to the statistical mechanics but also to the continuum mechanics. The statistical mechanics is mostly precise but is in fact too detailed and 
in many cases huge calculations crop up. The continuum mechanics is an asymptotic notion coming from short range interactions between molecules. It follows a loose of information but a more efficient and directly computable theory. In the simplest case of continuum mechanics, residual information comes through stress tensor like Cauchy tensor ${ }^{1,2}$. The concept of stress tensor is so frequently used that it has become as natural as the notion of force. Nevertheless, tensor of contact couples can be investigated as in Cosserat medium ${ }^{3}$ or configuration forces like in Gurtin approach ${ }^{4}$ with edge interactions of Noll and Virga ${ }^{5}$. Stress tensors and contact forces are interrelated notions ${ }^{6}$.

A fundamental point of view in continuum mechanics is: the Newton system for forces is equivalent to the work of forces is the value of a linear functional of displacements. Such a method due to Lagrange is dual of the system of forces due to Newton ${ }^{7,8}$ and is not issued from a variational approach; the minimization of the energy coincides with the functional approach in a special variational principle only for some equilibrium cases.

The linear functional expressing the work of forces is related to the theory of distributions; a decomposition theorem associated with displacements (as test functions whose supports are $C^{\infty}$ compact manifolds) uniquely determines a canonical zero order form (separated form) with respect both to the test functions and the transverse derivatives of contact test functions ${ }^{9}$. As Newton's principle is useless when we do not have any constitutive equation for the expression of forces, the linear functional method is useless when we do not have any constitutive assumption for the virtual work functional. The choice of the simple material theory associated with the Cauchy stress tensor corresponds with a constitutive assumption on its virtual work functional. It is important to notice that constitutive equations for the free energy $\chi$ and constitutive assumption for the virtual work functional may be incompatible ${ }^{10}$ : for any virtual displacement $\boldsymbol{\zeta}$ of an isothermal medium, the variation $-\delta \chi$ must be equal to the virtual work of internal forces $\delta \tau_{i n t}$. The equilibrium state is then obtained by the existence of a solution minimizing the free energy.

The equation of motion of a continuous medium is deduced from the d'Alembert-Lagrange principle of virtual works which is an extension of the principle in mechanics of systems with a finite number of degrees of freedom: The motion is such that for any virtual displacement the virtual work of forces is equal to the virtual work of mass accelerations.

Let us note: if the virtual work of forces is expressed in classical notations 
in the form

$$
\delta \tau=\iiint_{D}\{\mathbf{f} . \boldsymbol{\zeta}+\operatorname{tr}[(-p \mathbf{1}+2 \mu \nabla \mathbf{V}) \cdot \nabla \boldsymbol{\zeta}]\} d v+\iint_{S} \mathbf{T} . \boldsymbol{\zeta} d s
$$

from the d'Alembert-Lagrange principle, we obtain not only the equations of balance momentum for a viscous fluid in the domain $D$ but also the boundary conditions on the border $S$ of $D$. We notice that expression (1) is not the Frechet derivative of any functional expression.

If the free energy depends on the strain tensor $F$, then $\delta \tau$ must depend on $\nabla \boldsymbol{\zeta}$ and leads to the existence of the Cauchy stress tensor. If the free energy depends on the strain tensor $F$ and on the overstrain tensor $\nabla F$, then $\delta \tau$ must depend on $\nabla \boldsymbol{\zeta}$ and $\nabla^{2} \boldsymbol{\zeta}$.

Conjugated (or transposed) mappings being denoted by asterisk, for any vectors $\mathbf{a}, \mathbf{b}$, we write $\mathbf{a}^{*} \mathbf{b}$ for their scalar product (the line vector is multiplied by the column vector) and $\mathbf{a b}^{*}$ or $\mathbf{a} \otimes \mathbf{b}$ for their tensor product (the column vector is multiplied by the line vector). The product of a mapping $\mathbf{A}$ by a vector $\mathbf{a}$ is denoted by $\mathbf{A} \mathbf{a}$. Notation $\mathbf{b}^{*} \mathbf{A}$ means the covector $\mathbf{c}^{*}$ defined by the rule $\mathbf{c}^{*}=\left(\mathbf{A}^{*} \mathbf{b}\right)^{*}$. The divergence of a linear transformation $\mathbf{A}$ is the covector $\operatorname{div} \mathbf{A}$ such that, for any constant vector $\mathbf{a},(\operatorname{div} \mathbf{A}) \mathbf{a}=\operatorname{div}(\mathbf{A} \mathbf{a})$. We introduce a Galilean or fixed system of coordinates $\left(x^{1}, x^{2}, x^{3}\right)$ which is also denoted by $\mathbf{x}$ as Euler or spatial variables. If $f$ is a real function of $\mathbf{x}$, $\frac{\partial f}{\partial \mathbf{x}}$ is the linear form associated with the gradient of $f$ and $\frac{\partial f}{\partial x^{i}}=\left(\frac{\partial f}{\partial \mathbf{x}}\right)_{i}$; consequently, $\left(\frac{\partial f}{\partial \mathbf{x}}\right)^{*}=\operatorname{grad} f$. The identity tensor is denoted by $\mathbf{1}$.

Now, we present the method and its consequences in different cases of gradient theory. As examples, we revisit the case of Laplace theory of capillarity and the case of van der Waals fluids.

\section{Virtual work of continuous medium}

The motion of a continuous medium is classically represented by a continuous transformation $\varphi$ of a three-dimensional space into the physical space. In order to describe the transformation analytically, the variables $\mathbf{X}=\left(X^{1}, X^{2}, X^{3}\right)$ which single out individual particles correspond to material or Lagrange variables. Then, the transformation representing the motion of a continuous medium is

$$
\mathbf{x}=\varphi(\mathbf{X}, t) \quad \text { or } x^{i}=\varphi^{i}\left(X^{1}, X^{2}, X^{3}, t\right), i=1,2,3
$$

where $t$ denotes the time. At $t$ fixed the transformation possesses an inverse and continuous derivatives up to the second order except at singular sur- 
faces, curves or points. Then, the diffeomorphism $\varphi$ from the set $D_{0}$ of the particles into the physical space $D$ is an element of a functional space $\wp$ of the positions of the continuous medium considered as a manifold with an infinite number of dimensions.

To formulate the d'Alembert-Lagrange principle of virtual works, we introduce the notion of virtual displacements. This is obtained by letting the displacements arise from variations in the paths of the particles. Let a one-parameter family of varied paths or virtual motions denoted by $\left\{\varphi_{\eta}\right\}$ and possessing continuous derivatives up to the second order and expressed analytically by the transformation

$$
\mathbf{x}=\mathbf{\Phi}(\mathbf{X}, t ; \eta)
$$

with $\eta \in O$, where $O$ is an open real set containing 0 and such that $\boldsymbol{\Phi}(\mathbf{X}, t ; 0)=\varphi(\mathbf{X}, t)$ or $\varphi_{0}=\varphi$ (the real motion of the continuous medium is obtained when $\eta=0$ ). The derivation with respect to $\eta$ when $\eta=0$ is denoted by $\delta$. Derivation $\delta$ is named variation and the virtual displacement is the variation of the position of the medium ${ }^{11}$. The virtual displacement is a tangent vector to $\wp$ in $\varphi\left(\delta \varphi \in T_{\varphi}(\wp)\right)$. In the physical space, the virtual displacement $\delta \varphi$ is determined by the variation of each particle: the virtual displacement of the particle $\mathbf{x}$ is such that $\zeta=\delta \mathbf{x}$ when $\delta \mathbf{X}=0$, $\delta \eta=1$ at $\eta=0$; we associate the field of tangent vectors to $D$

$$
\mathbf{x} \in D \rightarrow \boldsymbol{\zeta}=\left.\psi(\mathbf{x}) \equiv \frac{\partial \Phi}{\partial \eta}\right|_{\eta=0} \in T_{\mathbf{x}}(D)
$$

where $T_{\mathbf{x}}(D)$ is the tangent vector bundle to $D$ at $\mathbf{x}$. The concept of virtual
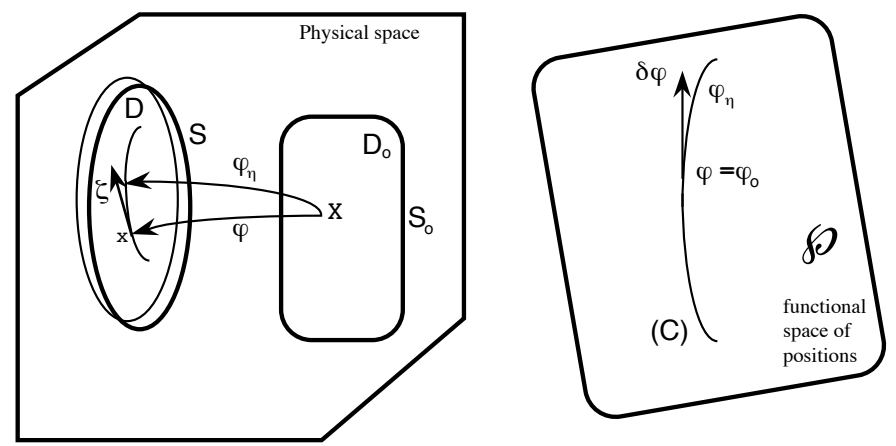

Fig. 1. The boundary $S$ of $D$ is represented by a thick curve and its variation by a thin curve. Variation $\delta \varphi$ of family $\left\{\varphi_{\eta}\right\}$ of varied paths belongs to $T_{\varphi}(\wp)$, tangent space to $(\wp)$ at $\varphi$. 
work is purposed in the form:

The virtual work is a linear functional value of the virtual displacement,

$$
\delta \tau=\langle\Im, \delta \varphi>
$$

where $\langle., .>$ denotes the inner product of $\Im$ and $\delta \varphi$; then, $\Im$ belongs to the cotangent space of $\wp$ at $\varphi\left(\Im \in T_{\varphi}^{*}(\wp)\right)$.

In Relation (2), the medium in position $\varphi$ is submitted to the covector $\Im$ denoting all the stresses; in the case of motion, we must add the inertial forces associated with the acceleration quantities to the volume forces. The d'Alembert-Lagrange principle of virtual works is expressed as:

For all virtual displacements, the virtual work is null. Consequently, representation (2) leads to:

$$
\forall \delta \varphi \in T_{\varphi}(\wp), \delta \tau=0
$$

Theorem: If expression (2) is a distribution in a separated form, the d'Alembert-Lagrange principle yields the equations of motions and boundary conditions in the form $\Im=0$.

\section{Some examples of linear functional of forces}

Among all possible choices of linear functional of virtual displacements, we classify the following ones:

\subsection{Model of zero gradient}

\subsubsection{Model A.0}

The medium fills an open set $D$ of the physical space and the linear functional is in the form

$$
\delta \tau=\iiint_{D} F_{i} \zeta^{i} d v
$$

where $F_{i}(i=1,2,3)$ denote the covariant components of the volume force $\mathbf{F}$ (including the inertial force terms) presented as a covector. The equation of the motion is

$$
\forall \mathbf{x} \in D, \quad F_{i}=0 \Leftrightarrow \mathbf{F}=0
$$

\subsubsection{Model B.0}

The medium fills a set $D$ and the surface $S$ is the boundary of $D$ belonging to the medium; with the same notations as in section 3.1.1, the linear 
functional is in the form

$$
\delta \tau=\iiint_{D} F_{i} \zeta^{i} d v+\iint_{S} T_{i} \zeta^{i} d s
$$

$T_{i}$ are the components of the surface forces (tension) T. From Eq. (4), we obtain the equation of motion as in Eq. (3) and the boundary condition,

$$
\forall \mathbf{x} \in S, T_{i}=0 \Leftrightarrow \mathbf{T}=0
$$

\subsection{Model of first gradient}

\subsubsection{Model A.1}

With the previous notations, the linear functional is in the form

$$
\delta \tau=\iiint_{D}\left(F_{i} \zeta^{i}-\sigma_{i}^{j} \zeta_{, j}^{i}\right) d v
$$

where $\sigma_{i}^{j}(i, j=1,2,3)$ are the components of the stress tensor $\sigma$. Stokes formula gets back to the model B.O in the separated form

$$
\delta \tau=\iiint_{D}\left(F_{i}+\sigma_{i, j}^{j}\right) \zeta^{i} d v-\iint_{S} n_{j} \sigma_{i}^{j} \zeta^{i} d s
$$

where $n_{j}(j=1,2,3)$ are the components of a covector which is the annulator of the vectors belonging to the tangent plane at the boundary $S$. It is not necessary to have a metric in the physical space; nevertheless, for the sake of simplicity it is convenient to use the Euclidian metric; the vector $\mathbf{n}$ of components $n^{j}(j=1,2,3)$ represents the external normal to $S$ relatively to $D$; the covector $\mathbf{n}^{\star}$ is associated with the components $n_{j}$. We deduce the equation of motion

$$
\forall \mathbf{x} \in D, \quad F_{i}+\sigma_{i, j}^{j}=0 \Leftrightarrow \mathbf{F}+\operatorname{div} \sigma=0
$$

and the boundary condition

$$
\forall \mathbf{x} \in S, \quad n_{j} \sigma_{i}^{j}=0 \Leftrightarrow \mathbf{n}^{\star} \sigma=0
$$

\subsubsection{Model B.1/0: (Mixed model with first gradient in $D$ and zero gradient on $S$ )}

The linear functional is expressed in the form

$$
\delta \tau=\iiint_{D}\left(F_{i} \zeta^{i}-\sigma_{i}^{j} \zeta_{, j}^{i}\right) d v+\iint_{S} T_{i} \zeta^{i} d s
$$


Stokes formula yields the separated form

$\delta \tau=\iiint_{D}\left(F_{i}+\sigma_{i, j}^{j}\right) \zeta^{i} d v+\iint_{S}\left(T_{i}-n_{j} \sigma_{i}^{j}\right) \zeta^{i} d s$

and we deduce the equation of motion in the same form as Eq. (5) and the boundary condition

$$
\forall \mathbf{x} \in S, \quad n_{j} \sigma_{i}^{j}=T_{i} \Leftrightarrow \mathbf{n}^{\star} \sigma=\mathbf{T}
$$

Model B.1/O is the classical theory for elastic media and fluids in continuum mechanics.

\subsubsection{Model B.1}

The linear functional is expressed in the form

$$
\delta \tau=\iiint_{D}\left(F_{i} \zeta^{i}-\sigma_{i}^{j} \zeta_{, j}^{i}\right) d v+\iint_{S}\left(T_{i} \zeta^{i}+\gamma_{i}^{j} \zeta_{, j}^{i}\right) d s
$$

where the tensor $\gamma$ of components $\gamma_{i}^{j}$ is a new term. The boundary of $D$ is a surface $S$ shared in a partition of $N$ parts $S_{p}$ of class $C^{2},(p=1, \ldots, N)$ (Fig. 2). We denote by $\left(R_{m}\right)^{-1}$ the mean curvature of $S$; the edge $\Gamma_{p}$ of $S_{p}$ is the union of the limit edges $\Gamma_{p q}$ between surfaces $S_{p}$ and $S_{q}$ assumed to be of class $C^{2}$ and $\mathbf{t}$ is the tangent vector to $\Gamma_{p}$ oriented by $\mathbf{n} ; \mathbf{n}^{\prime}$ is the unit external normal vector to $\Gamma_{p}$ in the tangent plane to $S_{p}: \mathbf{n}^{\prime}=\mathbf{t} \times \mathbf{n}$. Let us notice that:

$$
\gamma_{i}^{j} \zeta_{, j}^{i}=-\gamma_{i, j}^{j} \zeta^{i}+V_{, j}^{j}
$$

where $V^{j}=\gamma_{i}^{j} \zeta^{i}$; consequently, from integration of the divergence of

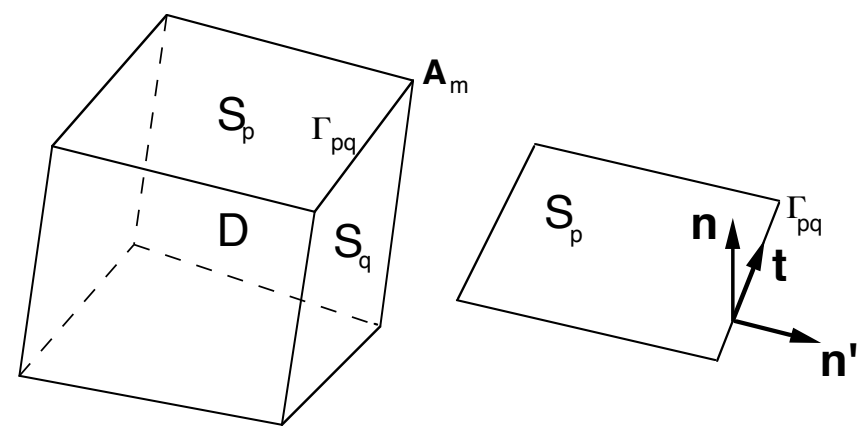

Fig. 2. The set $D$ has a surface boundary $S$ divided in several parts. The edge of $S$ is denoted by $\Gamma$ which is also divided in several parts with end points $\mathbf{A}_{m}$. 
vector $\mathbf{V}$ on surfaces $S_{p}$ we obtain,

$$
\iint_{S_{p}} V_{, j}^{j} d s=-\iint_{S_{p}} n_{j}\left(\frac{V^{j}}{R_{m}}-V_{, l}^{j} n^{l}\right) d s+\int_{\Gamma_{p}} n_{j}^{\prime} V^{j} d \ell
$$

We emphasize with the fact that $V_{l}^{j} n^{l}$ corresponds to the normal derivative to $S_{p}$ denoted $\frac{d V^{j}}{d n}$. An integration by parts of the term $\sigma_{i}^{j} \zeta_{, j}^{i}$ in relation (6) and taking account of relations (7-8) implies

$$
\delta \tau=\iiint_{D} F_{i}^{1} \zeta^{i} d v+\iint_{S} T_{i}^{1} \zeta^{i} d s+\iint_{S} L_{i} \frac{d \zeta^{i}}{d n} d s+\sum_{p=1}^{N} \int_{\Gamma_{p}} R_{p i} \zeta^{i} d \ell
$$

with the following definitions

$$
\begin{cases}F_{i}^{1} \equiv F_{i}+\sigma_{i, j}^{j}, & L_{i} \equiv n_{j} \gamma_{i}^{j} \\ T_{i}^{1} \equiv T_{i}-n_{j}\left(\sigma_{i}^{j}-\frac{d}{d n}\left(\gamma_{i}^{j}\right)+\frac{1}{R_{m}} \gamma_{i}^{j}\right)-\gamma_{i, j}^{j}, & R_{p i} \equiv n_{j}^{\prime} \gamma_{i}^{j}\end{cases}
$$

Due to theorem $37 \mathrm{in}^{9}$, the distribution (S.1) has a unique decomposition in displacements and transverse derivatives of displacements on the manifolds associated with D and its boundaries: expression $(S .1)$ is in a separated form. Consequently, the equation of motion is

$$
\forall \mathbf{x} \in D, F_{i}^{1}=0 \Leftrightarrow \mathbf{F}^{1}=0
$$

and the boundary conditions are

$$
\begin{array}{ll}
\forall \mathbf{x} \in S, T_{i}^{1}=0, L_{i}=0 \Leftrightarrow & \mathbf{T}^{1}=0, \mathbf{L}=0 \\
\forall \mathbf{x} \in \Gamma_{p q}, R_{p i}+R_{q i}=0 \Leftrightarrow & \mathbf{R}_{p}+\mathbf{R}_{q}=0
\end{array}
$$

Term $\mathbf{L}$ is not reducible to a force: its virtual work $L_{i} \frac{d \zeta^{i}}{d n}$ is not the product of a force with the displacement $\boldsymbol{\zeta}$; the term $\mathbf{L}$ is an embedding action.

\subsection{Model of second gradient}

3.3.1. Model A.2

The linear functional is in the form

$$
\delta \tau=\iiint_{D}\left(F_{i} \zeta^{i}-\sigma_{i}^{j} \zeta_{, j}^{i}+S_{i}^{j k} \zeta_{, j k}^{i}\right) d v
$$

Tensor $\mathbf{S}$ with $S_{i}^{j k}=S_{i}^{k j}$ is an overstress tensor. An integration by parts of the last term brings back to the model B.1,

$$
\delta \tau=\iiint_{D}\left(F_{i} \zeta^{i}-\left(\sigma_{i}^{j}+S_{i, k}^{j k}\right) \zeta_{, j}^{i}\right) d v+\iint_{S} n_{k} S_{i}^{j k} \zeta_{, j}^{i} d s
$$


and the virtual work gets the separated form $(S .1)$ with:

$$
\left\{\begin{array}{cc}
F_{i}^{1}=F_{i}+\sigma_{i, j}^{j}+S_{i, j k}^{j k} & \text { volume force } \\
T_{i}^{1}=-n_{j}\left(\sigma_{i}^{j}+S_{i, k}^{j k}-\frac{d}{d n}\left(n_{k} S_{i}^{j k}\right)+\frac{1}{R_{m}} n_{k} S_{i}^{j k}\right) & \text { surface force } \\
R_{p i}=n_{j}^{\prime} n_{k} S_{i}^{j k} & \text { line force } \\
L_{i}=n_{j} n_{k} S_{i}^{j k} & \text { embedding action }
\end{array}\right.
$$

and consequently yields the same equation of motion and boundary conditions as in case B.1.

\subsubsection{Model B.2}

The linear functional is in the form

$\delta \tau=\iiint_{D}\left(F_{i} \zeta^{i}-\sigma_{i}^{j} \zeta_{, j}^{i}+S_{i}^{j k} \zeta_{, j k}^{i}\right) d v+\iint_{S}\left(T_{i} \zeta^{i}+\gamma_{i}^{j} \zeta_{, j}^{i}+U_{i}^{j k} \zeta_{, j k}^{i}\right) d s$

This functional yields two integrations successively on $S_{p}$ and on $\Gamma_{p q}$ with terms at the points $A_{m}$. With obvious notations, for the same reasons as in section 3.2.3, the virtual work gets the separated form

$$
\begin{aligned}
\delta \tau= & \iiint_{D} F_{i}^{1} \zeta^{i} d v+\iint_{S}\left(T_{i}^{1} \zeta^{i}+L_{i}^{1} \frac{d \zeta^{i}}{d n}+L_{i}^{2} \frac{d^{2} \zeta^{i}}{d n^{2}}\right) d s \\
& +\sum_{p} \int_{\Gamma_{p}}\left(R_{p i} \zeta^{i}+M_{p i} \frac{d \zeta^{i}}{d n^{\prime}}\right) d \ell+\sum_{m} \phi_{m i} \zeta_{\mathbf{A}_{m}}^{i}
\end{aligned}
$$

where $\zeta_{\mathbf{A}_{m}}^{i}(i=1,2,3)$ are the components of $\boldsymbol{\zeta}$ at point $\mathbf{A}_{m}$. The calculations are not expended. They introduce the curvature tensor on $S_{p}$ and the geodesic curvature of $\Gamma_{p q .}$. Consequently, $F_{i}^{1}, T_{i}^{1}, R_{p i}, \phi_{m i}$ are associated with volume, surface, line and forces at points; $L_{i}^{1}, L_{i}^{2}, M_{p i}$ are embedding efforts of order 1 and 2 on $S$ and of order 1 on the edge $\Gamma$. The equation of motion and boundary conditions express that these seven tensorial quantities are null on their domains of values $D, S, \Gamma_{p}$ and $\mathbf{A}_{m}$.

\section{Conclusion}

It is possible to extend the previous presentation by means of more complex medium with gradient of order $n$. The models introduce embedding effects of more important order on surfaces, edges and points. The (A.n) model refers to a (B.n-1) model: the fact that boundary surface $S$ is (or is not) a material surface has now a physical meaning. Consequently, we can resume the previous presentation as follows: 
a) The choice of a model corresponds to specify the part $G$ of the algebraic dual $T_{\varphi}^{*}(\wp)$ in which the efforts are considered: $\Im \in G \subset T_{\varphi}^{*}(\wp)$.

b) In order to operate with the principle of virtual works and to obtain the mechanical equations in the form $\Im=0$, it is no matter that the part $G$ of the dual is separating $(\forall \Im \in G,<\Im, \delta \varphi>=0 \Rightarrow \delta \varphi=0)$, but it is important the part $G$ is separated $\left(\Im \in G, \forall \delta \varphi \in T_{\varphi}(\wp),<\Im, \delta \varphi>=0 \Rightarrow\right.$ $\Im=0)$.

c) The functionals $A .1, B .1 / 0, A .2, B .2$ are not separated: if $\Im$ consists in the data of the fields $\mathbf{F}, \sigma, \mathbf{T}$, it is not possible to conclude that the fields are zero.

d) Functionals in A.0, B.0, S.1, S.2 ... are separated: if the fields $\mathbf{S}^{1}, \mathbf{T}^{1}$, $\mathbf{R}^{1}, \mathbf{L}, \ldots$ are continuous then, by using the fundamental lemma of variation calculus, their values must be equal to zero. They are the only functionals we must know for using the principle of virtual works; it is exactly as for a solid: the torque of forces is only known in the equations of motion.

e) When the fields are not continuous on surfaces or curves, we have to consider a model of greater order in gradients and to introduce integrals on inner boundaries of the medium.

For conservative medium, the first gradient theory corresponds to the compressible case. The theory of fluid, elastic, viscous and plastic media refers to the model $(S . O)$. The Laplace theory of capillarity in fluids refers to the model $(S .1)$. To take into account superficial effects acting between solids and fluids, we use the model of fluids endowed with capillarity (S.2); the theory interprets the capillarity in a continuous way and contains the Laplace theory of capillarity; for solids, the model corresponds to "elastic materials with couple stresses" indicated by Toupin in ${ }^{12}$.

\section{Example 1: The Laplace theory of capillarity}

Liquid-vapor and two-phase interfaces are represented by a material surface endowed with an energy relating to Laplace surface tension. The interface appears as a surface separating two media with its own characteristic behavior and energy properties ${ }^{13}$ (when working far from critical conditions, the capillary layer has a thickness equivalent to a few molecular beams ${ }^{14}$ ). The Laplace theory of capillarity refers to the model B.1 in the form $(S .1)$ as following: for a compressible fluid with a capillary effect on the wall boundaries, the free energy is in the form

$$
\chi=\iiint_{D} \rho \alpha(\rho) d v+\sum_{p=1}^{N} a_{p} \iint_{S_{p}} d s
$$


where $\alpha(\rho)$ is the fluid specific energy, $\rho$ is the matter density and coefficients $a_{p}$ are the surface tensions of each surface $S_{p}$. Surface integrations are associated to the space metric; the virtual work of internal forces is

$\delta \tau_{\text {int }}=\iiint_{D}-p_{, i} \zeta^{i} d v+\sum_{p=1}^{N} \iint_{S_{p}} n_{i}\left(p-\frac{a_{p}}{R_{m}}\right) \zeta^{i} d s+\sum_{p=1}^{N} \int_{\Gamma_{p}} a_{p} n_{i}^{\prime} \zeta^{i} d \ell$ where $p \equiv \rho^{2} \alpha^{\prime}(\rho)$ is the fluid pressure. The external force (including inertial forces) is the body force $\rho \mathbf{f}$ defined in $D$, the surface force is $\mathbf{T}$ defined on $S$ and the line force is $\mathbf{R}$ defined on $\Gamma$. D'Alembert-Lagrange principle yields the equation of motion and boundary conditions:

$$
\begin{gathered}
\forall \mathbf{x} \in D,-p_{, i}+\rho f_{i}=0 \quad \Leftrightarrow \quad-\operatorname{grad} p+\rho \mathbf{f}=0, \\
\forall \mathbf{x} \in S, \quad p n_{i}+T_{i}-a_{p} \frac{n_{i}}{R_{m}}=0 \quad \Leftrightarrow \quad p \mathbf{n}+\mathbf{T}-a_{p} \frac{\mathbf{n}}{R_{m}}=0 \\
\forall \mathbf{x} \in \Gamma_{p q}, \quad a_{p} n_{p i}^{\prime}+a_{q} n_{q i}^{\prime}+R_{i}=0
\end{gathered}
$$

Boundary conditions are Laplace equation and Young-Dupré condition.

\section{Example 2: Fluids endowed with internal capillarity}

For interfacial layers, kinetic theory of gas leads to laws of state associated with non-convex internal energies ${ }^{15,16}$. This approach dates back to van der Waals $^{17}$, Korteweg ${ }^{18}$, corresponds to the Landau-Ginzburg theory ${ }^{19}$ and presents two disadvantages. First, between phases, the pressure may become negative; simple physical experiments can be used to cause traction that leads to these negative pressure values ${ }^{20}$. Second, in the field between bulks, internal energy cannot be represented by a convex surface associated with density and entropy; this fact seems to contradict the existence of equilibrium states; it is possible to eliminate this disadvantage by writing in an anisotropic form the stress tensor of the capillary layer which allows to study interfaces of non-molecular size near a critical point.

One of the problems that complicates this study of phase transformation dynamics is the apparent contradiction between Korteweg classical stress theory and the Clausius-Duhem inequality ${ }^{21}$. Proposal made by Eglit ${ }^{22}$, Dunn and Serrin ${ }^{23}$, Casal and Gouin ${ }^{24}$ and others rectifies this anomaly for liquid-vapor interfaces. The simplest model in continuum mechanics considers a free energy as the sum of two terms: a first one corresponding to a medium with a uniform composition equal to the local one and a second one associated with the non-uniformity of the fluid ${ }^{15,17}$. The second term is approximated by a gradient expansion, typically truncated to 
the second order. The model is simpler than models associated with the renormalization-group theory ${ }^{25}$ but has the advantage of easily extending well-known results for equilibrium cases to the dynamics of interfaces ${ }^{26-28}$. We consider a fluid $D$ in contact with a wall $S$. Physical experiments prove that the fluid is nonhomogeneous in the neighborhood of $S^{16}$. The internal energy $\varepsilon$ is also a function of the entropy. In the case of isothermal motions, the internal energy is replaced by the free energy. In the mechanical case, the entropy and the temperature are not concerned by the virtual displacements of the medium. Consequently, for isentropic or isothermal motions, $\varepsilon=f(\rho, \beta)$ where $\beta=(\operatorname{grad} \rho)^{2}$. The fluid is submitted to external forces represented by a potential $\Omega$ as a function of Eulerian variables $\mathbf{x}$. To obtain boundary conditions it is necessary to know the wall effect. An explicit form for the energy of interaction between surfaces and liquids is proposed $\mathrm{in}^{29}$. We denote by $B$ the surface density of energy at the wall. The total energy $E$ of the fluid is the sum of three potential energies: $E_{f}$ (bulk energy), $E_{p}$ (external energy) and $E_{S}$ (surface energy).

$$
E_{f}=\iiint_{D} \rho \varepsilon(\rho, \beta) d v, E_{p}=\iiint_{D} \rho \Omega(\mathbf{x}) d v, E_{S}=\iint_{S} B d s
$$

We have the results (see Appendix): $\delta E_{f}=\iiint_{D}(-\operatorname{div} \sigma) \zeta d v$

$$
+\iint_{S}\left\{-A \frac{d \zeta_{n}}{d n}+\left(\frac{2 A}{R_{m}} \mathbf{n}^{*}+\operatorname{grad}_{t g}^{*} A+\mathbf{n}^{*} \sigma\right) \boldsymbol{\zeta}\right\} d s-\int_{\Gamma} A \mathbf{n}^{\prime *} \boldsymbol{\zeta} d \ell
$$

with $\sigma=-P \mathbf{1}-C \operatorname{grad} \rho \otimes \operatorname{grad} \rho \equiv-P \mathbf{1}-C\left(\frac{\partial \rho}{\partial \mathbf{x}}\right)^{*} \frac{\partial \rho}{\partial \mathbf{x}}$, where $C=2 \rho \varepsilon_{\beta}^{\prime}$, $P=\rho^{2} \varepsilon_{\rho}^{\prime}-\rho \operatorname{div}(C \operatorname{grad} \rho), \varepsilon_{\rho}^{\prime}\left(\right.$ or $\left.\varepsilon_{\beta}^{\prime}\right)$ denoting the partial derivative of $\varepsilon$ with respect to $\rho$ (or $\beta), \zeta_{n}=\mathbf{n}^{*} \boldsymbol{\zeta} ; A=C \rho \frac{d \rho}{d n}$ where $\frac{d \rho}{d n}=\frac{\partial \rho}{\partial \mathbf{x}} \mathbf{n}$ and $\operatorname{grad}_{t g}$ denotes the tangential part of the gradient relatively to $S$.

$$
\begin{aligned}
\delta E_{p} & =\iiint_{D} \rho \frac{\partial \Omega}{\partial \mathbf{x}} \boldsymbol{\zeta} d v \equiv \iiint_{D} \rho\left(\operatorname{grad}^{*} \Omega\right) \boldsymbol{\zeta} d v ; \text { and }, \\
\delta E_{S} & =\iint_{S}\left\{\delta B-\left(\frac{2 B}{R_{m}} \mathbf{n}^{*}+\operatorname{grad}_{t g}^{*} B\right) \zeta\right\} d s+\int_{\Gamma} B \mathbf{n}^{*} \boldsymbol{\zeta} d \ell
\end{aligned}
$$

The density in the fluid has a limit value $\rho_{S}$ at the wall $S$ and $B$ is assumed to be a function of $\rho_{S}$ only $^{29}$. Then, $\delta B=B^{\prime}\left(\rho_{S}\right) \delta \rho_{S}=-\rho_{S} B^{\prime}\left(\rho_{S}\right) \operatorname{div} \boldsymbol{\zeta}$, where $\operatorname{div} \zeta$ is computed on $S^{11}$. Let us denote $G=-\rho_{S} B^{\prime}\left(\rho_{S}\right)$; Appendix yields

$$
\iint_{S} \delta B d s=\iint_{S} G \operatorname{div} \zeta d s=
$$




$$
\begin{gathered}
=\iint_{S}\left\{G \frac{d \zeta_{n}}{d n}-\left(\frac{2 G}{R_{m}} \mathbf{n}^{*}+\operatorname{grad}_{t g}^{*} G\right) \boldsymbol{\zeta}\right\} d s+\int_{\Gamma} G \mathbf{n}^{*} \boldsymbol{\zeta} d \ell \\
\delta E_{S}=\iint_{S}\left\{G \frac{d \zeta_{n}}{d n}-\left(\frac{2 H}{R_{m}} \mathbf{n}^{*}+\operatorname{grad}_{t g}^{*} H\right) \boldsymbol{\zeta}\right\} d s+\int_{\Gamma} H \mathbf{n}^{\prime *} \boldsymbol{\zeta} d \ell
\end{gathered}
$$

with $H=B\left(\rho_{S}\right)-\rho_{S} B^{\prime}\left(\rho_{S}\right)$. Then,

$$
\begin{aligned}
& \delta E=\iiint_{D}\left(\rho \operatorname{grad}^{*} \Omega-\operatorname{div} \sigma\right) \boldsymbol{\zeta} d v-\int_{\Gamma}(A-H) \mathbf{n}^{\prime *} \boldsymbol{\zeta} d \ell \\
& +\iint_{S}(G-A) \frac{d \zeta_{n}}{d n}+\left(\frac{2(A-H)}{R_{m}} \mathbf{n}^{*}+\operatorname{grad}_{t g}^{*}(A-H)+\mathbf{n}^{*} \sigma\right) \boldsymbol{\zeta} d s
\end{aligned}
$$

At equilibrium, $\delta \tau \equiv-\delta E=0$. The fundamental lemma of variation calculus associated with separated form (9) corresponding to (S.2), yields:

\subsection{Equation of equilibrium}

From any arbitrary variation $\mathrm{x} \in D \rightarrow \boldsymbol{\zeta}(\mathbf{x})$ such that $\boldsymbol{\zeta}=\mathbf{0}$ on $S$, we get

$$
\begin{gathered}
\iiint_{D}\left(\rho \operatorname{grad}^{*} \Omega-\operatorname{div} \sigma\right) \zeta d s=0 . \text { Then, } \\
\rho \operatorname{grad}^{*} \Omega-\operatorname{div} \sigma=0
\end{gathered}
$$

This equation is written in the classical form of equation of equilibrium ${ }^{24}$. It is not the same for the boundary conditions.

\subsection{Boundary conditions}

\subsubsection{Case of a rigid wall}

We consider a rigid wall; on $S$, the virtual displacements satisfy the condition $\mathbf{n}^{*} \boldsymbol{\zeta}=0$. Then, at the rigid wall, $\forall \mathbf{x} \in S \rightarrow \boldsymbol{\zeta}(\mathbf{x})$ such that $\mathbf{n}^{*} \boldsymbol{\zeta}=0$,

$$
\iint_{S}(G-A) \frac{d \zeta_{n}}{d n}+\left(\frac{2(A-H)}{R_{m}} \mathbf{n}^{*}+\operatorname{grad}_{t g}^{*}(A-H)+\mathbf{n}^{*} \sigma\right) \boldsymbol{\zeta} d s=0
$$

Due to $\sigma=\sigma^{*}$, we deduce the boundary conditions (11-12)

$$
\forall \mathbf{x} \in S, G-A=0,
$$

and there exists a Lagrange multiplier $\mathbf{x} \in S \rightarrow \lambda(\mathbf{x}) \in R$ such that,

$$
\forall \mathbf{x} \in S, \frac{2(A-H)}{R_{m}} \mathbf{n}+\operatorname{grad}_{t g}(A-H)+\sigma \mathbf{n}=\lambda \mathbf{n}
$$

The edge $\Gamma$ of $S$ belongs to the solid wall and consequently on $\Gamma, \boldsymbol{\zeta}=\eta \mathbf{t}$ : the integral on $\Gamma$ is null and does not yield any additive condition. 


\subsubsection{Case of an elastic wall}

The equilibrium equation (10) is unchanged. On $S$, the condition (11) is also unchanged. The only different condition comes from the fact that we do not have anymore the slipping condition for the virtual displacement on $S,\left(\mathbf{n}^{*} \boldsymbol{\zeta}=0\right)$. Due to the possible deformation of the wall, the virtual work of stresses on $S$ is $\delta E_{e}=\iint_{S} \boldsymbol{\kappa}^{*} \boldsymbol{\zeta} d s+\int_{\Gamma} \mathbf{R}^{*} \boldsymbol{\zeta} d \ell$ where $\boldsymbol{\kappa}=\sigma_{e} \mathbf{n}$ is the stress (loading) vector associated with stress tensor $\sigma_{e}$ of the elastic wall and $\mathbf{R}$ is the line force due to the elasticity of the line. Relation (12) is replaced by

$$
\forall \mathbf{x} \in S, 2 \frac{(A-H)}{R_{m}} \mathbf{n}+\operatorname{grad}_{t g}(A-H)+\sigma \mathbf{n}+\boldsymbol{\kappa}=0
$$

We obtain an additive condition on $\Gamma$ in the form $(H-A) \mathbf{n}^{\prime}+\mathbf{R}=0$ and due to condition (11),

$$
\forall \mathbf{x} \in \Gamma, B \mathbf{n}^{\prime}+\mathbf{R}=0
$$

(If $\Gamma$ is the union of edges $\Gamma_{p}, B \mathbf{n}^{\prime}$ is replaced by $\sum_{p} B_{p} \mathbf{n}_{p}^{\prime}$ ).

\subsection{Analysis of the boundary conditions}

Eq. (11) yields $C \frac{d \rho}{d n}+B^{\prime}\left(\rho_{S}\right)=0$; the definition of $\sigma$ implies:

$$
\sigma \mathbf{n}=-P \mathbf{n}-C \frac{d \rho}{d n} \operatorname{grad} \rho
$$

Due to the fact that the tangential part of Eq. (12) is always verified, the only condition comes from Eq. (11); Eq. (12) yields the value of the Lagrange multiplier $\lambda$ and Eq. (13) the value of $\mathbf{R}$. For an elastic (nonrigid) wall we obtain,

$$
\kappa_{t g}=0 \quad \text { and } \quad \kappa_{n}=P+\frac{2 B}{R_{m}}-B^{\prime}\left(\rho_{S}\right) \frac{d \rho}{d n}
$$

where $\kappa_{t g}$ and $\kappa_{n}$ are the tangential and the normal components of $\boldsymbol{\kappa}$. Taking into account of Eq. (14) we obtain the stress values at the non rigid elastic wall. The surface energy is ${ }^{29}: B\left(\rho_{S}\right)=-\gamma_{1} \rho_{S}+\frac{\gamma_{2}}{2} \rho_{S}^{2}$ where $\gamma_{1}$ and $\gamma_{2}$ are two positive constants and the fluid density condition at the wall is

$$
C \frac{d \rho}{d n}=\gamma_{1}-\gamma_{2} \rho_{S}
$$


If we denote by $\rho_{B}=\gamma_{1} / \gamma_{2}$ the bifurcation fluid density at the wall, due to the fact $C$ is positive constant ${ }^{16}$, we obtain: if $\rho_{S}<\rho_{B}$, (or $\left.\rho_{S}>\rho_{B}\right), \frac{d \rho}{d n}$ is positive (or negative) and we have a lack (or excess) of fluid density at the wall. Such media allow to study fluid interfaces and interfacial layers between fluids and solids and lead to numerical and asymptotic methods ${ }^{30}$. The extension to the dynamic case is straightforward: Eq. (10) yields

$$
\rho \Gamma^{*}-\operatorname{div} \sigma+\rho \operatorname{grad}^{*} \Omega=0
$$

Vector $\boldsymbol{\Gamma}$ is the acceleration; boundary conditions (11-14) are unchanged.

\section{Acknowledgments}

I am grateful to Professor Tommaso Ruggeri for helpful discussions.

\section{Appendix}

Let $S$ be a surface in the 3 -dimensional space and $\mathbf{n}$ its external normal extended locally in the vicinity of $S$ by the expression $\mathbf{n}(\mathbf{x})=\operatorname{grad} d(\mathbf{x})$, where $d$ is the distance of a point $\mathbf{x}$ to $S$; for any vector field $\mathbf{w}$, we obtain ${ }^{31}$ :

$$
\operatorname{rot}(\mathbf{n} \times \mathbf{w})=\mathbf{n} \operatorname{div} \mathbf{w}-\mathbf{w} \operatorname{div} \mathbf{n}+\frac{\partial \mathbf{n}}{\partial \mathbf{x}} \mathbf{w}-\frac{\partial \mathbf{w}}{\partial \mathbf{x}} \mathbf{n}
$$

From $\mathbf{n}^{*} \frac{\partial \mathbf{n}}{\partial \mathbf{x}}=0$ and $\operatorname{div} \mathbf{n}=-\frac{2}{R_{m}}$ we deduce on $S$,

$$
\mathbf{n}^{*} \operatorname{rot}(\mathbf{n} \times \mathbf{w})=\operatorname{div} \mathbf{w}+\frac{2}{R_{m}} \mathbf{n}^{*} \mathbf{w}-\mathbf{n}^{*} \frac{\partial \mathbf{w}}{\partial \mathbf{x}} \mathbf{n}
$$

We deduce: for any scalar field $A$ and $\mathbf{w}=A \boldsymbol{\zeta}$,

$$
A \operatorname{div} \boldsymbol{\zeta}=A \frac{d \zeta_{n}}{d n}-\frac{2 A}{R_{m}} \zeta_{n}-\left(\operatorname{grad}_{t g}^{*} A\right) \boldsymbol{\zeta}+\mathbf{n}^{*} \operatorname{rot}(A \mathbf{n} \times \boldsymbol{\zeta})
$$

Let us calculate $\delta E_{f} ; D$ is a material volume, then $\delta E_{f}=\iiint_{D} \rho \delta \varepsilon d v$ with $\delta \varepsilon=\frac{\partial \varepsilon}{\partial \rho} \delta \rho+\frac{\partial \varepsilon}{\partial \beta} \delta \beta$. From $\delta \frac{\partial \rho}{\partial \mathbf{x}}=\frac{\partial \delta \rho}{\partial \mathbf{x}}-\frac{\partial \rho}{\partial \mathbf{x}} \frac{\partial \zeta}{\partial \mathbf{x}} \quad\left(\right.$ see $\left.^{24}\right)$,

$$
\begin{gathered}
\rho \varepsilon_{\beta}^{\prime} \delta \beta=2 \rho \varepsilon_{\beta}^{\prime} \delta \frac{\partial \rho}{\partial \mathbf{x}}\left(\frac{\partial \rho}{\partial \mathbf{x}}\right)^{*}=C\left(\frac{\partial \delta \rho}{\partial \mathbf{x}}-\frac{\partial \rho}{\partial \mathbf{x}} \frac{\partial \boldsymbol{\zeta}}{\partial \mathbf{x}}\right)\left(\frac{\partial \rho}{\partial \mathbf{x}}\right)^{*} \\
=\operatorname{div}(C \operatorname{grad} \rho \delta \rho)-\operatorname{div}(C \operatorname{grad} \rho) \delta \rho-\operatorname{tr}\left(C \operatorname{grad} \rho \operatorname{grad}^{*} \rho \frac{\partial \boldsymbol{\zeta}}{\partial \mathbf{x}}\right)
\end{gathered}
$$


Due to $\delta \rho=-\rho \operatorname{div} \zeta\left(\right.$ see $\left.^{11}\right)$,

$$
\begin{gathered}
\delta E_{f}=\iiint_{D}\left(\frac{\partial P}{\partial \mathbf{x}}+\operatorname{div}\left(C \operatorname{grad} \rho \operatorname{grad}^{*} \rho\right)\right) \boldsymbol{\zeta} d v \\
-\iiint_{D} \operatorname{div}\left(C \rho \operatorname{grad} \rho \operatorname{div} \boldsymbol{\zeta}+C \operatorname{grad} \rho \operatorname{grad}^{*} \rho \boldsymbol{\zeta}+P \boldsymbol{\zeta}\right) d v \\
=\iiint_{D}-(\operatorname{div} \sigma) \boldsymbol{\zeta} d v+\iint_{S}\left(-A \operatorname{div} \boldsymbol{\zeta}+\mathbf{n}^{*} \sigma \boldsymbol{\zeta}\right) d s
\end{gathered}
$$

From Eq. (16), we deduce immediatly: $\delta E_{f}=\iiint_{D}(-\operatorname{div} \sigma) \boldsymbol{\zeta} d v+$

$$
\iint_{S}\left\{-A \frac{d \zeta_{n}}{d n}+\left(\frac{2 A}{R_{m}} \mathbf{n}^{*}+\operatorname{grad}_{t g}^{*} A+\mathbf{n}^{*} \sigma\right) \boldsymbol{\zeta}\right\} d s-\int_{\Gamma} A \mathbf{n}^{\prime *} \boldsymbol{\zeta} d \ell
$$

Let us calculate $\delta E_{S}$; due to $E_{S}=\iint_{S} B \operatorname{det}\left(\mathbf{n}, d_{1} \mathbf{x}, d_{2} \mathbf{x}\right)$ where $d_{1} \mathbf{x}$ and $d_{2} \mathbf{x}$ are two coordinate lines of $S$, we get:

$$
E_{S}=\iint_{S_{0}} B \operatorname{det} F \operatorname{det}\left(F^{-1} \mathbf{n}, d_{1} \mathbf{X}, d_{2} \mathbf{X}\right)
$$

where $S_{0}$ is the image of $S$ in a reference space with Lagrangian coordinates $\mathbf{X}$ and $F$ is the deformation gradient tensor $\frac{\partial \mathbf{x}}{\partial \mathbf{X}}$ of components $\left\{\frac{\partial x^{i}}{\partial X^{j}}\right\}$.

Then, $\quad \delta E_{S}=\iint_{S_{0}} \delta B \operatorname{det} F \operatorname{det}\left(F^{-1} \mathbf{n}, d_{1} \mathbf{X}, d_{2} \mathbf{X}\right)$

$$
+\iint_{S_{0}} B \delta\left(\operatorname{det} F \operatorname{det}\left(F^{-1} \mathbf{n}, d_{1} \mathbf{X}, d_{2} \mathbf{X}\right)\right) \text {. }
$$

with $\iint_{S_{0}} B \delta\left(\operatorname{det} F \operatorname{det}\left(F^{-1} \mathbf{n}, d_{1} \mathbf{X}, d_{2} \mathbf{X}\right)\right)=$

$\iint_{S} B \operatorname{div} \boldsymbol{\zeta} \operatorname{det}\left(\mathbf{n}, d_{1} \mathbf{x}, d_{2} \mathbf{x}\right)+B \operatorname{det}\left(\frac{\partial \mathbf{n}}{\partial \mathbf{x}} \boldsymbol{\zeta}, d_{1} \mathbf{x}, d_{2} \mathbf{x}\right)$
$-B \operatorname{det}\left(\frac{\partial \zeta}{\partial \mathbf{x}} \mathbf{n}, d_{1} \mathbf{x}, d_{2} \mathbf{x}\right)=\iint_{S}\left(\operatorname{div}(B \boldsymbol{\zeta})-\left(\operatorname{grad}^{*} B\right) \boldsymbol{\zeta}-B \mathbf{n}^{*} \frac{\partial \boldsymbol{\zeta}}{\partial \mathbf{x}} \mathbf{n}\right) d s$

Relation (15) yields: $\operatorname{div}(B \boldsymbol{\zeta})+\frac{2 B}{R_{m}} \mathbf{n}^{*} \boldsymbol{\zeta}-\mathbf{n}^{*} \frac{\partial B \boldsymbol{\zeta}}{\partial \mathbf{x}} \mathbf{n}=\mathbf{n}^{*} \operatorname{rot}(B \mathbf{n} \times \boldsymbol{\zeta})$,

$$
\begin{gathered}
\iint_{S_{0}} B \delta\left(\operatorname{det} F \operatorname{det}\left(F^{-1} \mathbf{n}, d_{1} \mathbf{X}, d_{2} \mathbf{X}\right)\right)= \\
\iint_{S_{0}}\left(-\frac{2 B}{R_{m}} \mathbf{n}^{*}+\operatorname{grad}^{*} B\left(\mathbf{n n}^{*}-\mathbf{1}\right)\right) \boldsymbol{\zeta} d s+\iint_{S} \mathbf{n}^{*} \operatorname{rot}(B \mathbf{n} \times \boldsymbol{\zeta}) d s
\end{gathered}
$$

where $\operatorname{grad}^{*} B\left(\mathbf{n n}^{*}-\mathbf{1}\right)$ belongs to the cotangent plane to $S$; we obtain

$$
\delta E_{S}=\iint_{S}\left(\delta B-\left(\frac{2 B}{R_{m}} \mathbf{n}^{*}+\operatorname{grad}_{t g}^{*} B\right) \boldsymbol{\zeta}\right) d s+\int_{\Gamma} B \mathbf{n}^{\prime *} \boldsymbol{\zeta} d \ell .
$$




\section{References}

1. P. Germain, Cours de mécanique des milieux continus (Masson, Paris, 1973).

2. C. Truesdell, First course in rational continuum mechanics (Academic Press, New York, 1994).

3. E. Cosserat and F. Cosserat, Sur la théorie des corps déformables (Hermann, Paris, 1909).

4. M.E. Gurtin, Configurational forces as basic concepts in continuum physics (Springer, Berlin, 2000).

5. W. Noll and E.G. Virga, Arch. Rat. Mech. Anal. 3, 1 (1990).

6. F. dell'Isola and P. Seppecher, Meccanica 32, 33 (1997).

7. P. Germain, J. Mécanique, 12, 235 (1973).

8. P. Germain, S.I.A.M. J. Appl. Math. 25, 556 (1973).

9. L. Schwartz, Théorie des distributions, Ch. 3 (Hermann, Paris, 1966).

10. H. Gouin and F. Gouin, Mech. Res. Comm. 10, 21 (1983).

11. J. Serrin, Mathematical principles of classical fluid mechanics, in Encyclopedia of Physics VIII/1, Ed: S. Flügge, (Springer, Berlin, 1960) pp. 125-263.

12. R.A. Toupin, Arch. Rat. mech. Anal. 11, 385 (1962).

13. V. Levitch, Physicochemical Hydrodynamics (Prentice-Hall, New Jersey, 1962).

14. S. Ono and S. Kondo, Molecular theory of surface tension in liquid, in Encyclopedia of Physics, X, Ed: S. Flügge (Springer, Berlin, 1960).

15. J.W. Cahn and J.E. Hilliard, J. Chem. Phys. 31, 688 (1959).

16. J.S. Rowlinson. and B. Widom, Molecular theory of capillarity (Clarendon Press, Oxford, 1984).

17. J.D. van der Waals, Archives Néerlandaises 28, 121 (1894-1895).

18. J. Korteweg, Archives Néerlandaises 2, $\mathbf{n}^{\circ}$ 6, 1 (1901).

19. P.C. Hohenberg and B.I. Halperin, Rev. Mod. Phys. 49, 435 (1977).

20. Y. Rocard, Thermodynamique (Masson, Paris, 1952).

21. M.E. Gurtin, Arch. Rat. Mech. Anal. 19, 339 (1965).

22. M.E. Eglit, J. Appl. Math. Mech. 29, 351 (1965).

23. J.E. Dunn and J. Serrin, Arch. Rat. Mech. Anal. 88, 95 (1985).

24. P. Casal and H. Gouin, C. R. Acad. Sci. Paris 300, II, 231 (1985).

25. C. Domb, The critical point (Taylor \& Francis, London, 1996).

26. M. Slemrod, Arch. Rat. Mech. Anal. 81, 301 (1983).

27. L. Truskinovsky, P.M.M. 51, 777 (1987).

28. H. Gouin and T. Ruggeri, Eur. J. Mech B/ Fluids 24, 596 (2005).

29. H. Gouin, J. Phys. Chem. B 102, 1212 (1998).

30. H. Gouin and S.L. Gavrilyuk, Physica A, 268, 291 (1999).

31. S. Kobayashi and K. Nomizu, Foundations of differential geometry, vol. 1 (Interscience Publ., New York, 1963). 